\title{
Warfarin combined with low dose aspirin in myocardial infarction did not provide clinical benefit beyond that of aspirin alone
}

Fiore LD, Ezekowitz MD, Brophy MT, et al., for the Combination Hemotherapy and Mortality Prevention (CHAMP) Study Group. Department of Veterans Affairs Cooperative Studies Program Clinical Trial comparing combined warfarin and aspirin with aspirin alone in survivors of acute myocardial infarction. Primary results of the CHAMP study. Circulation 2002 Feb 5;105:557-63.

\section{QUESTION: In patients who have survived an acute myocardial infarction (MI), is warfarin combined with aspirin more effective than aspirin alone?}

\section{Design}

Randomised (allocation concealed*), unblinded,* controlled trial with a median 2.7 years of follow up.

\section{Setting}

78 Department of Veterans Affairs medical centres in the US.

\section{Patients}

5059 patients (median age 62 y, 98\% men) who had an acute MI within the previous 14 days. Exclusion criteria included comorbid conditions that limited life expectancy to $<2$ years, ongoing bleeding, drug or alcohol dependence, and hypersensitivity to aspirin or warfarin. Vital status was obtained for $99 \%$ of the patients.

\section{Intervention}

2522 patients were assigned to warfarin (target international normalised ratio [INR] 1.5 to $2.5 \mathrm{IU}$ ) plus aspirin $(81 \mathrm{mg} / \mathrm{d})$, and 2537 were assigned to aspirin alone (162 mg/d).

\section{Main outcome measures}

The primary outcome was all-cause mortality. Secondary outcomes were recurrent MI, stroke, and major haemorrhage.

\section{Main results}

Analysis was by intention to treat. The median INR in the warfarin group was $1.8 \mathrm{IU}$. Groups did not differ for all cause mortality, recurrent MI, or stroke (table). The study had an $80 \%$ power for detecting a $15 \%$ reduction in annual mortality with combination treatment relative to that with aspirin alone. More major bleeding occurred in patients in the warfarin plus aspirin group than in those assigned to aspirin alone (1.28 $v 0.72$ events per 100 person y of follow up, $p<0.001)$. Intracranial haemorrhage rates were identical in the 2 groups (14 patients per treatment group).

\section{Conclusions}

In patients who have survived an acute myocardial infarction, warfarin combined with low dose aspirin did not provide a clinical benefit beyond that achieved with aspirin alone. Major bleeding occurred more frequently with combination treatment.

*See glossary.
Warfarin plus aspirin $(W+A) v$ aspirin alone $(A)$ in acute myocardial infarction $(M I)$ at a median of 2.7 yearst

\begin{tabular}{lllll} 
Outcomes & W+A & A & RRI $(95 \%$ CI $)$ & NNH \\
All cause mortality & $17.6 \%$ & $17.3 \%$ & $2 \%(-10$ to 15$)$ & Not significant \\
\hline Recurrent MI & $13.3 \%$ & $13.1 \%$ & $2 \%(-12$ to 17$)$ & Not significant \\
& & & RRR $(\mathrm{Cl})$ & NNT \\
Stroke & $3.1 \%$ & $3.5 \%$ & $11 \%(-20$ to 34$)$ & Not significant \\
\hline
\end{tabular}

†Abbreviations defined in glossary; RRI, RRR, NNH, NNT, and Cl calculated from data in article.

\section{COMMENTARY}

The long term use of low dose antiplatelet drugs (eg, aspirin and clopidogrel) to prevent recurrent infarction is established practice. Aiming to enhance survival after MI, some research groups have studied combined warfarin anticoagulation treatment with the antithrombotic action of aspirin.

The CHAMP study shows that combining low intensity warfarin anticoagulation treatment (median INR $<2.0$ ) with aspirin for about 3 years does not prolong life beyond the effect of aspirin alone, regardless of when the treatment was started after MI. Moreover, the combination decreased neither MI recurrence nor stroke. The results concur with a similar study in patients with acute coronary syndromes, which suggested better survival at 3 months only. Short term prophylaxis merits further study.

Could some subsets of patients receive particular benefit from a warfarin plus aspirin treatment? In the CHAMP study, the infarction location and left ventricular function did not affect total mortality. Bleeding risks increase with biological age, but we do not know at what age the risks for antithrombotic action begin to outweigh the survival gain; therefore, we cannot use age as a guide.

Combined treatment in the CHAMP study caused 1.7 times more major bleeding than did aspirin alone, without adding any further benefit. The careful patient monitoring done during a trial would be expected to minimise major bleeding; so this finding is probably an underestimate of the harm that would occur in usual clinical practice. The evidence argues against adding low intensity warfarin anticoagulation treatment to aspirin in patients who have survived MI.

A regimen adding moderate intensity (INR $>2$ ) or high intensity (INR >3) warfarin anticoagulation treatment to aspirin, with or without clopidogrel, will probably cause even more bleeding than antiplatelet treatment alone and offset any additional survival gain. ${ }^{1}$ Likewise, we need to weigh the haemorrhagic risk associated with such regimens as clopidogrel plus aspirin against their antithrombotic efficacy. Laboratory study evidence suggests that clopidogrel plus aspirin inhibits platelet aggregation more effectively than does aspirin alone. ${ }^{2}$ Furthermore, the combination was more effective than aspirin alone in unstable angina with non-ST segment elevation MI. ${ }^{3}$

Vernon M Oh, MD, FRCP National University Hospital Singapore, Republic of Singapore

1 Anand SS, Yusuf S, Poque J, et al. Long-term oral anticoagulant therapy in patients with unstable angina or suspected non-Q-wave myocardial infarction: organization to assess strategies for ischemic syndromes (OASIS) pilot study

2 Moshfegh K, Redondo; M, Julmy F, et al. Antiplatelet effects of clopidogrel compared with aspirin after myocardial infarction: enhanced inhibitory effects of combination therapy. $J$ Am Coll Cardiol 2000;36:699-705.

Yusuf S, Zhao F, Mehta SR, et al. Effects of clopidogrel in addition to aspirin in patients with acute coronary syndromes without ST-segment elevation. N Engl J Med
2001;345:494-502. 